\title{
Enhanced spatial resolution on figures versus grounds
}

\author{
Lauren N. Hecht ${ }^{1,4} \cdot$ Joshua D. Cosman ${ }^{2} \cdot$ Shaun P. Vecera ${ }^{3}$
}

Published online: 5 April 2016

(C) The Psychonomic Society, Inc. 2016

\begin{abstract}
Much is known about the cues that determine figure-ground assignment, but less is known about the consequences of figure-ground assignment on later visual processing. Previous work has demonstrated that regions assigned figural status are subjectively more shape-like and salient than background regions. The increase in subjective salience of figural regions could be caused by a number of processes, one of which may be enhanced perceptual processing (e.g., an enhanced neural representation) of figures relative to grounds. We explored this hypothesis by having observers perform a perceptually demanding spatial resolution task in which targets appeared on either figure or ground regions. To rule out a purely attentional account of figural salience, observers discriminated targets on the basis of a region's color (red or green), which was equally likely to define the figure or the ground. The results of our experiments showed that targets appearing on figures were discriminated more accurately than those appearing in ground regions. In addition, targets appearing on figures were discriminated better than those presented in regions considered figurally neutral, but targets appearing within ground regions were discriminated more poorly than those appearing in figurally neutral regions. Taken together, our findings suggest that when two regions share a contour, regions assigned as
\end{abstract}

Lauren N. Hecht

lhecht@gustavus.edu

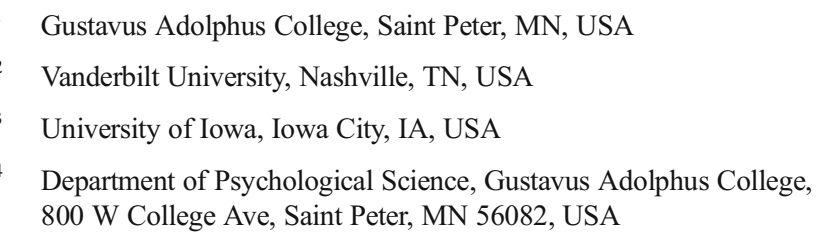

Department of Psychological Science, Gustavus Adolphus College, 800 W College Ave, Saint Peter, MN 56082, USA

figure are perceptually enhanced, whereas regions assigned as ground are perceptually suppressed.

Keywords Perceptual organization · Visual perception · Spatial vision

A fundamental property of the visual system is the ability to segregate objects from one another in a visual scene. For instance, when attempting to grab a book from a cluttered desktop, we are able to select and act upon the book with relative ease, despite the presence of other irrelevant objects. This ability to effectively segregate objects in a visual scene is the result of several visual processes, one of which is figureground assignment. During figure-ground assignment, candidate objects ("figures") are segregated from backgrounds, allowing us to process and act upon only a focused subset of the information present in a scene. This process is trivial in situations in which a single object is present on a uniform background; however, in most real-world visual scenes, we are confronted with a number of objects that overlap and partially occlude one another, making figure-ground assignment much more difficult. As a result, sophisticated visual mechanisms must be implemented to effectively select which regions are associated with a particular contour and, consequently, distinguish figures from grounds.

\section{Control of figure-ground assignment}

Beginning with the influential work of the Gestalt psychologists, research on figure-ground assignment has focused on the factors that control the segregation of candidate objects from their backgrounds. These cues to figure-ground assignment can be viewed as control settings that determine which 
regions in a scene are likely to be perceived as figures. Extensive evidence suggests that both bottom-up and topdown factors control figure-ground assignment (see Palmer, 1999, 2002, and Wagemans et al., 2012, for reviews).

Initial studies focused on the bottom-up control of figureground assignment. For example, Rubin (1915/1958) noted that area influences figure-ground assignment, in that smaller regions are more likely to be perceived as figure than are larger regions. Likewise, both symmetric regions (i.e., those with matching left and right parts; Bahnsen, 1928) and convex (i.e., outwardly bulging) regions are more likely to be viewed as figure (Kanizsa \& Gerbino, 1976; Pomerantz \& Kubovy, 1986; Rock, 1975). More recently, Vecera, Vogel, and Woodman (2002) showed that regions falling below a horizon line (i.e., the "lower region") of a scene are more likely to be perceived as figure than those falling above a horizon line. These stimulus-based cues are important because they are invariant properties of figures that can allow viewers to perform figure-ground assignment even when confronted with novel scenes or scenes containing novel objects. As a result, the stimulus properties themselves are often sufficient to drive figure-ground assignment, affording the visual system the flexibility required to make segregation processes efficient even in situations in which little or no top-down information is available (see Vecera \& O'Reilly, 2000).

In addition to the influence of stimulus properties on figure-ground assignment, there is evidence that top-down inputs control figure-ground assignment. For example, object familiarity influences which region of a display is perceived as figure (Peterson, 1994; Peterson \& Gibson, 1993; Rock, 1975). When observers view figure-ground stimuli that contain a familiar or meaningful region (e.g., a face in profile), they tend to see that region as figure. However, if that same display is rotated $180^{\circ}$, familiarity affects figure-ground assignment much less, if at all, suggesting that both top-down and image-based properties interact with one another during figure-ground assignment.

Other top-down processes also affect figure-ground assignment. For example, attending to a particular region of a display makes that region more likely to be perceived as figure (Driver \& Baylis, 1996; Vecera, Flevaris, \& Filapek, 2004). Furthermore, Vecera et al. (2004) provided evidence that when both image-based and top-down cues (convexity and attention, respectively) were present in the same display, these cues competed with one another to influence which region observers would see as figure. This combination of both topdown and bottom-up cues in the control of figure-ground assignment fits both with interactive accounts of figureground segregation (Vecera \& O'Reilly, 1998, 2000; see also Craft, Schütze, Niebur, \& von der Heydt, 2007; Roelfsema, Lamme, Spekreijse, \& Bosch, 2002) and with more general accounts of "biased competition" in visual processing (Desimone \& Duncan, 1995; see also Vecera, 2000).
Figure-ground assignment can thus be viewed as a competition between two regions for figural status, and various cues bias the figural assignment to one region over others.

\section{Consequences of figure-ground assignment}

As we outlined above, much is known about the factors that control figure-ground assignment. However, less is known about the possible consequences of this process on later visual processing. Phenomenologically, figures appear to be more shape-like and more salient than grounds, and there is evidence that the visual system processes figures differently from grounds, producing a "figural benefit" in different visual tasks. For instance, as was reported by Rubin (1915/1958), when viewing an ambiguous face/vase stimulus, holding the vase as figure makes it appear more distinct and shape-like than the opposing faces, which lack form and fall into the background (see also Koffka, 1935). Perhaps because figures appear more shape-like, they are more likely to be remembered, both in the long term (several minutes or longer; Rubin, 1915/1958; see also Dutton \& Traill, 1933; but see Rock \& Kremen, 1957) and in the short term (a few hundred milliseconds; Driver \& Baylis, 1996, Vecera et al., 2004). Although figures are processed differently than grounds, the underlying cause of this processing difference is unknown.

An early set of experiments by Wong and Weisstein (1982) examined the influence of figure-ground assignment on target detection. In their experiments, observers viewed Rubin's ambiguous face/vase stimulus and held either the vase or the faces as figure. Target detection was enhanced when the target fell within the region observers currently perceived as the "figure," suggesting a perceptual advantage for targets appearing in figure relative to background regions. Similarly, Nelson and Palmer (2007) showed that when observers viewed a figure-ground display in which one region contained a meaningful, or familiar, cue to figure-ground assignment (e.g., a face in profile), targets falling within this region were detected more quickly, likely because perceptual processing of these "figures" began prior to perceptual processing of grounds (Lester, Hecht, \& Vecera, 2009; see also Hecht, Spencer, \& Vecera, 2015). In addition to this effect of figural status on detection times, Nelson and Palmer also found better discrimination for targets appearing in figures.

One straightforward interpretation of the latter finding is that figure-ground assignment produces a perceptually enhanced representation of the figural region, which then allows target detection and discrimination to occur efficiently for targets on the figure. Perceptual enhancement could be a consequence of enhanced neural representation of figures, relative to ground regions, as has been predicted by neurophysiological studies and models of figure-ground assignment (e.g., Craft et al., 2007; Roelfsema et al., 2002; Vecera, 2000; 
Vecera \& O'Reilly, 1998, 2000). However, from the previous studies outlined above, it is unclear whether the behavioral processing benefits observed for targets appearing on figures are due to perceptual enhancement of the regions assigned as figure or to the attentional effects of figure-ground assignment. In other words, it is possible that these previously reported figural benefits are the result of attention being drawn to figures, rather than benefits related to figure-ground assignment per se. Such a possibility is plausible, because a number of studies have demonstrated perceptual processing benefits for items appearing at attended locations (see Carrasco, 2006, 2011, for reviews).

Thus, our focus in the present work was to determine whether or not figure-ground assignment increases the perceptibility of the figure, making the figure and targets appearing within it more perceptually salient, or whether the previously found enhancement effects were the result of attentional processes resulting from figure-ground assignment. We developed a task in which observers employed an attentional set for color (à la Folk, Remington, \& Johnston, 1992) to direct attention to one region of a multicolor display, and we manipulated figure-ground assignment orthogonally to this attention setting by using image-based convexity cues. More specifically, observers performed a perceptually demanding spatial resolution task, by responding to targets within a specific colored region (red, green) that was equally likely to be the figure (convex) or ground (concave) region (Fig. 1). Observers reported the spatial offset of two vertices (see Baylis \& Driver, 1993; Gibson, 1994). By manipulating the size of the offset of the relevant stimuli, we could measure perceptual sensitivity and assess the effects of figure-ground assignment on perceptual-level processing. Since attention should be drawn strongly to the region matching the observers' attentional set for color (Folk et al., 1992), regardless of that region's figural status, any benefit in spatial resolution for figures should result primarily from the perceptual

\section{a}

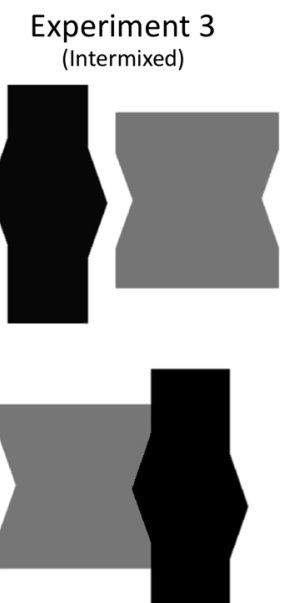

\section{b}
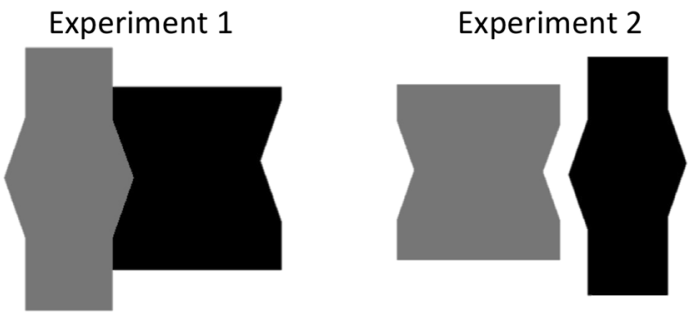

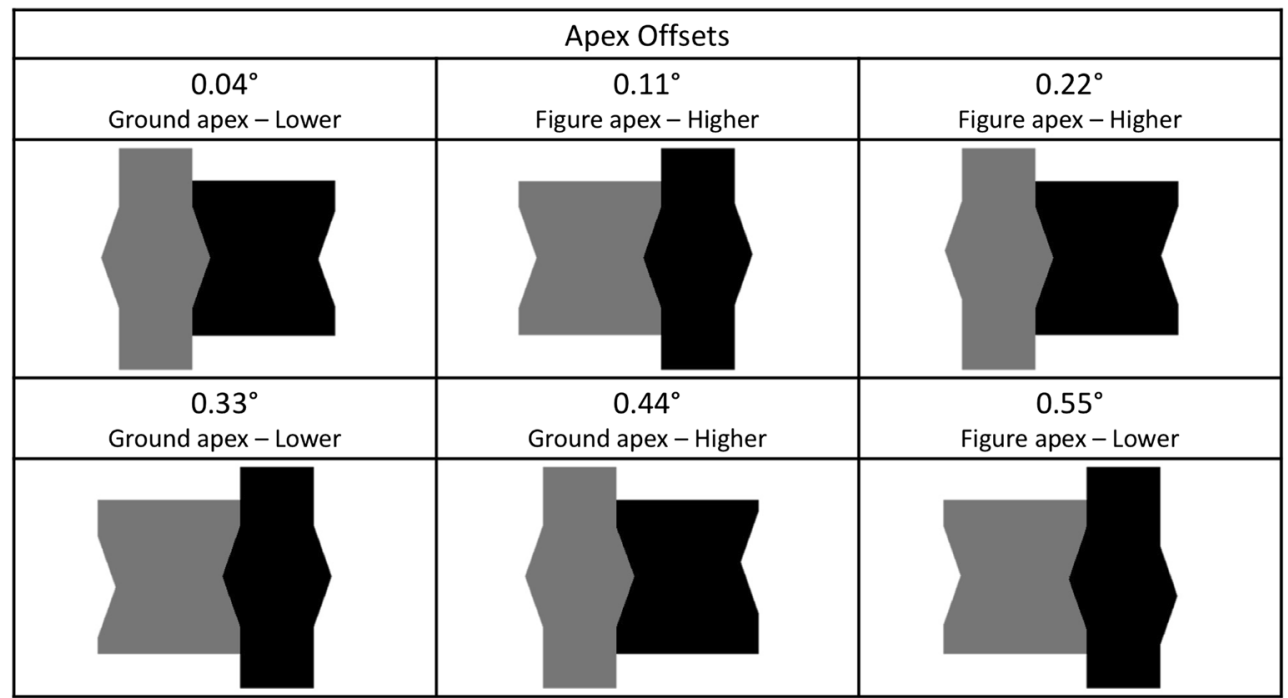

Fig. 1 a Examples of the stimuli used in each experiment. Figure position (left, right) and color (red, green) were fully counterbalanced. b Samples of apex offsets. The shifted apex was either higher or lower than the central (fixed) apex 
enhancement of figures relative to grounds. By the same logic, if no difference in spatial resolution performance were found between figures and grounds, the figural benefits observed in previous studies were likely due to attention and not to perceptual enhancement.

\section{Experiment 1}

\section{Method}

Participants Nineteen University of Iowa undergraduates received course credit for volunteering; all reported having normal or corrected-to-normal vision. This experiment complied with all ethical standards, as enforced by the University of Iowa's Institutional Review Board.

Stimuli The figure-ground displays, shown in Fig. 1, consisted of two regions and were designed such that one region of the display was clearly figural in nature, on the basis of image-based convexity cues (see Baylis \& Driver, 1993). One region was red and the other was green, with each color being equally likely to appear on the left or the right and to define either the figure (convex) or ground (concave) region. The ground region measured $4.83^{\circ} \times 5.05^{\circ}$ of visual angle and contained two concave angles $\left(135^{\circ}\right)$ that were separated by $2.5^{\circ}$ of visual angle. The ground region shared a contour with a figure (subtending $3.35^{\circ} \times 7.77^{\circ}$ of visual angle) containing two convex angles (also $135^{\circ}$ ) that were also separated by $2.5^{\circ}$ of visual angle. The position of the innermost apex that was shared by the two regions was held at a constant location in the center of the display (i.e., at fixation), whereas either of the two outermost apices (either that on the figure or that on the ground) was offset $0.04^{\circ}, 0.11^{\circ}, 0.22^{\circ}, 0.33^{\circ}, 0.44^{\circ}$, or $0.55^{\circ}$ of visual angle above or below this fixed apex (see Fig. 1b). This design allowed us to obtain a measure of observers' spatial resolution by having them make positional judgments of one of the outermost apices (either that on the green region or that on the red region) relative to the fixed central apex. The stimuli were viewed from a distance of approximately $77 \mathrm{~cm}$, and from this distance each display subtended approximately $7.91^{\circ} \times 7.77^{\circ}$ of visual angle.

We presented a total of 48 figure-ground displays: 2 color assignments (figure red, figure green) $\times 2$ positions (figure left, figure right) $\times 2$ offset directions (above fixed apex, below fixed apex $) \times 6$ angle offsets $\left(0.04^{\circ}, 0.11^{\circ}, 0.22^{\circ}, 0.33^{\circ}\right.$, $\left.0.44^{\circ}, 0.55^{\circ}\right)$.

Procedure The sequence and timing of events are depicted in Fig. 2. Each trial began with a fixation point, which was visible for $500 \mathrm{~ms}$. The figure-ground display was then presented for $200 \mathrm{~ms}$, too brief a duration for observers to make eye movements. The screen then remained blank until observers

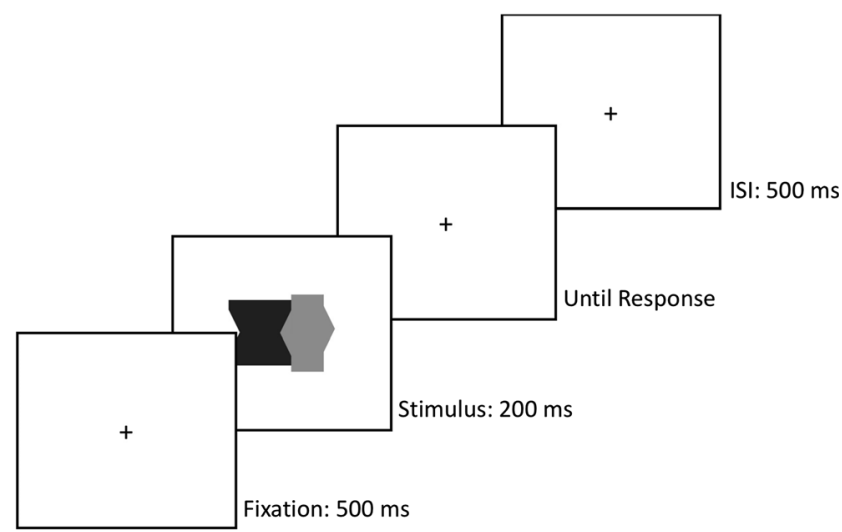

Fig. 2 Sequence and timing of events for a single trial in each experiment

had made a response, with a 500-ms intertrial interval preceding the presentation of the next trial.

In order to fully counterbalance both color and response, each observer responded in one of four ways during the spatial resolution task. First, observers attended to either the red or green region of the figure-ground display, according to the experimenter's instructions; half of the observers attended the red region of the display, and the other half attended the green region of the display throughout the experiment. Within each color condition, observers indicated which of the two apices, that on the right of the region or that on the left of the region, was either higher or lower; in other words, half of the observers reported which apex in the attended color region was higher, and half reported which apex was lower. For instance, an observer in the "green-higher" condition would attend the green region of the display and respond which of the apices (that on the left or right of the green region) was higher relative to the other. Observers responded using a button box, with the left index finger on the button for "left" responses and the right index finger on the button for "right" responses. Each observer received a block of 96 practice trials, which were not analyzed, followed by eight blocks of 96 trials.

\section{Results and discussion}

The data analyses include only observers with response accuracies significantly above chance (defined as $60 \%$, in this study). This criterion resulted in the exclusion of three participants from the final analysis, leaving data from 16 participants ( $n=4$ in each response condition) in the following analyses.

We analyzed the accuracy data with a two-factor analysis of variance (ANOVA), with Figural Status (apices on figure or ground) and Apex Offset $\left(0.04^{\circ}, 0.11^{\circ}, 0.22^{\circ}, 0.33^{\circ}, 0.44^{\circ}\right.$, or $\left.0.55^{\circ}\right)$ as factors. These data appear in Fig. 3. We observed a significant main effect of apex offset, $F(5,75)=21.6, p<$ $.0001, \eta_{\mathrm{p}}{ }^{2}=.59$, with larger offsets being discriminated more accurately than smaller offsets. Observers discriminated 


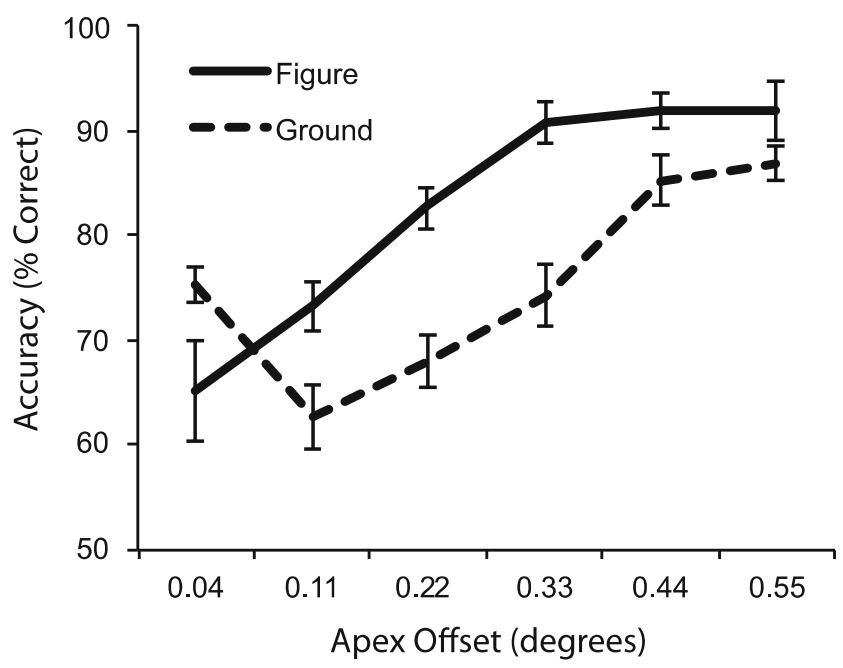

Fig. 3 Results of Experiment 1. Points represent the mean accuracy data for targets appearing in figure and ground regions, as a function of apex offset. Error bars represent 95\% within-subjects confidence intervals

targets falling in the figural region consistently more accurately than those falling in the ground region $(82.5 \%$ vs. $75.3 \%$, respectively), as evidenced by the significant main effect of figural status, $F(1,15)=8.01, p=.01, \eta_{\mathrm{p}}{ }^{2}=.35$. This main effect was accompanied by a significant two-way interaction, $F(5,75)=10.9, p<.001, \eta_{\mathrm{p}}{ }^{2}=.42$, indicating that spatial discriminability across the differing offsets was greater for figures than for grounds. We conducted planned comparisons to examine the figural benefit at each of the apex offsets. Planned comparisons showed that observers discriminated the offset of the apices significantly more accurately when the apices appeared on figures relative to grounds at all but the smallest $\left(0.04^{\circ}\right)$ and largest $\left(0.55^{\circ}\right)$ offsets (all significant $t \mathrm{~s}>2.4, p \mathrm{~s}<.02)$, possibly due to a floor and a ceiling effect, respectively.

These results suggest that figural status affected performance in our spatial resolution task. Given that we controlled for attention by requiring observers to adopt a consistent attentional set for color, the greater accuracy for spatial resolution judgments in figural than in ground regions appears to be the result of a perceptual enhancement effect. As such, it appears that regions assigned figural status possess an enhanced perceptual representation over regions assigned as grounds.

Although our results are consistent with an enhancement account, it is possible that the improved performance for targets appearing on figures is not the result of figure-ground assignment per se, but is instead due to a physical stimulus confound. Specifically, on trials in which the target appeared within the figure, observers compared points on two convexities, whereas on trials in which the target appeared on the ground, they compared points on two concavities. Thus, in Experiment 2 we separated the two regions of our display, such that they no longer shared a contour and thus appeared as two figures on a larger background. If the increased performance for targets appearing on figures results from an enhanced representation of the figure during figureground assignment, we would expect no difference in performance depending on which region of the display the target appeared in. However, if our results were instead due to a confound between figure-ground status and the convexity/ concavity of the shape on which the discrimination was performed, we would expect results identical to those observed in Experiment 1.

\section{Experiment 2}

\section{Method}

Participants Sixteen University of Iowa undergraduates received course credit for volunteering; all reported having normal or corrected-to-normal vision. This experiment complied with all ethical standards, as enforced by the University of Iowa's Institutional Review Board.

Stimuli The stimuli were identical to those used in Experiment 1, with the following exceptions: The two regions were separated by $0.44^{\circ}\left(0.22^{\circ}\right.$ on either side of fixation), appearing as two figures on a larger background. These separated displays subtended $8.20^{\circ} \times 7.77^{\circ}$ of visual angle.

Procedure The procedure was identical to that of Experiment 1.

\section{Results and discussion}

The accuracy data are shown in Fig. 4. These data were analyzed using a two-way ANOVA identical to that used in Experiment 1. As in Experiment 1, we observed a significant main effect of apex offset, $F(5,75)=38.9, p<.0001, \eta_{\mathrm{p}}{ }^{2}=$ .72 , with larger offsets being discriminated more accurately than smaller offsets. However, in contrast to Experiment 1, we did not observe a significant main effect related to which region contained the target, $F(1,15)<1$. This suggests that the results of Experiment 1 were not due to the shapes of the regions themselves, but instead to an enhancement of regions assigned figural status.

However, another alternative interpretation of our results must also be addressed. Specifically, it is possible that rather than being due to an enhancement of figural regions, the results of Experiments 1 and 2 were driven by a suppression of ground regions (Peterson \& Skow, 2008; Salvagio, Cacciamani, \& Peterson, 2012). Furthermore, if figureground assignment represents a competitive process (Vecera \& O'Reilly, 1998, 2000), it may be the case that both figure enhancement and ground suppression contributed to the 


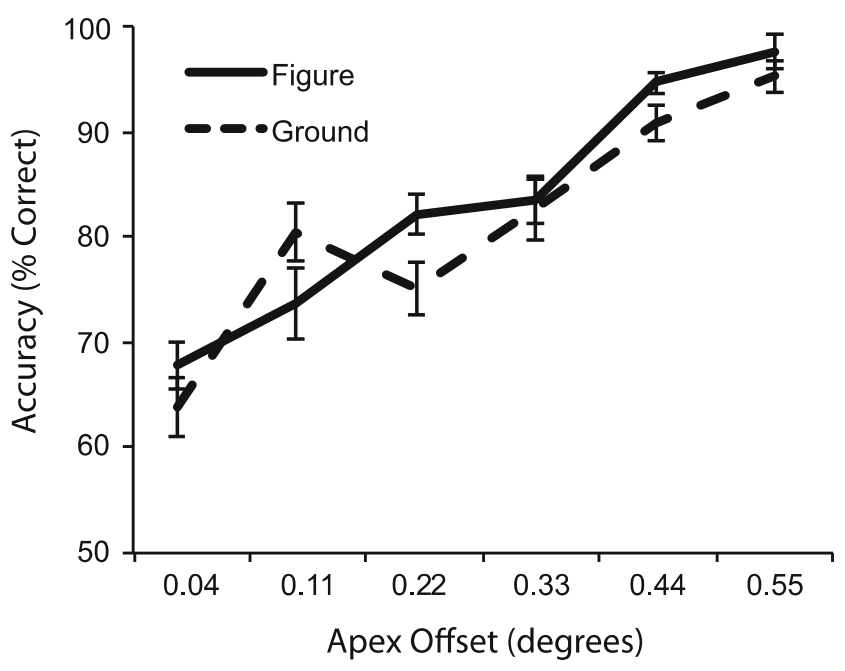

Fig. 4 Results of Experiment 2. Points represent the mean accuracy data for targets appearing in figure and ground regions, as a function of apex offset. Error bars represent $95 \%$ within-subjects confidence intervals

results above. In order to adjudicate between these possible mechanistic accounts, in Experiment 3 we interspersed the figure-ground displays from Experiment 1 with the figurally "neutral" displays used in Experiment 2, in which the objects no longer shared a contour, and thus should not show competitive influences with one another (Salvagio et al., 2012; Vecera \& O'Reilly, 2000).

Thus, if the effects observed in Experiment 1 were due solely to figural enhancement, in Experiment 3 we would expect to find no difference in performance for targets on neutral stimuli or ground regions, but a benefit for targets appearing on figures. Conversely, if the effect was driven by ground suppression, performance would decrease for targets on ground regions relative to equivalent performance for targets on neutral stimuli and figures. A combination of figural enhancement and ground suppression would lead to discrimination accuracy for targets appearing on neutral stimuli falling somewhere in-between those for targets appearing on figures versus grounds.

\section{Experiment 3}

\section{Method}

Participants Sixteen University of Iowa undergraduates received course credit for volunteering; all reported having normal or corrected-to-normal vision. This experiment complied with all ethical standards, as enforced by the University of Iowa's Institutional Review Board.

Stimuli The stimuli were identical to those used in Experiments 1 and 2, with the following exception:
Separated displays (Exp. 2) were interspersed with the figure-ground stimuli (Exp. 1).

Procedure The procedure was identical to those in Experiments 1 and 2, except for the following modifications. To keep the number of trials identical to that in the previous experiments, we only used three offsets $\left(0.04^{\circ}, 0.33^{\circ}\right.$, and, $\left.0.55^{\circ}\right)$. In half of the trials, the target appeared within either the "figure" region or the "ground" region of the stimuli used in Experiment 1. In the other half of the trials, the target appeared in one of the two (convex or concave) figurally "neutral" regions (formerly the figure and ground regions, respectively) of the stimuli used in Experiment 2. The sequence of events and timing were identical to those in the other experiments, with a 96-trial practice block (which was not analyzed) being followed by eight testing blocks of 96 trials each.

\section{Results and discussion}

The accuracy data are shown in Fig. 5. These data were analyzed using a two-way ANOVA similar to those used in the previous experiments, with the exception that figural status now had three levels (apices on figure, ground, or "neutral" region), and apex offset also had only three levels $\left(0.04^{\circ}\right.$, $0.33^{\circ}$, or $0.55^{\circ}$ ). As in the previous experiments, we observed a significant main effect of apex offset, $F(2,30)=156.2, p<$ $.0001, \eta_{\mathrm{p}}{ }^{2}=.91$, with larger offsets being discriminated more accurately than smaller offsets across all stimulus types. As in Experiment 1, we also observed a significant main effect of figural status, $F(2,30)=18.4, p<.0001, \eta_{\mathrm{p}}{ }^{2}=.55$, and an interaction between figural status and offset, $F(4,60)=3.52$, $p=.01, \eta_{\mathrm{p}}^{2}=.19$.

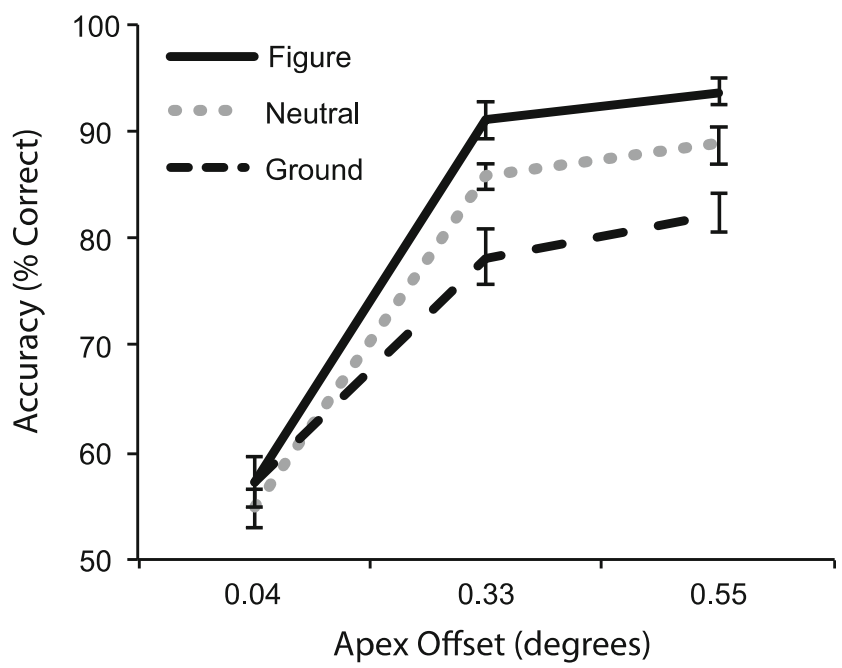

Fig. 5 Results of Experiment 3. Points represent the mean accuracy data for targets appearing in convex and concave regions, as a function of apex offset. Error bars represent 95\% within-subjects confidence intervals 
As can be seen in Fig. 5, we observed a significant benefit to spatial resolution accuracy when the target appeared in the figure region, as opposed to when it appeared in either the ground or neutral regions, at all but the smallest offset, replicating the results of Experiment 1 and providing support for a perceptual-enhancement account of figure-related processing benefits. This was confirmed by planned comparisons that showed a significant benefit in accuracy for targets appearing on figure relative to neutral regions $\left(0.33^{\circ}\right.$ offset: $t=2.67, p=$ $.02 ; 0.55^{\circ}$ offset: $\left.t=3.12, p<.01\right)$. However, we also observed a significant cost to accuracy when the target appeared in the ground region relative to either the figure or a neutral region, suggesting a concurrent perceptual suppression of ground regions, consistent with previous work (Peterson \& Skow, 2008; Salvagio et al., 2012). This was confirmed by planned comparisons that showed a significant decrement in accuracy for targets appearing on ground relative to neutral regions $\left(0.33^{\circ}\right.$ offset: $t=3.37, p<.01 ; 0.55^{\circ}$ offset: $t=2.88, p=.01$ ).

Thus, when observers viewed interspersed figure-ground and figurally neutral displays, discrimination accuracy was highest for the targets falling within figural regions, lowest for targets falling within ground regions, and intermediate for targets falling in neutral regions. This provides direct evidence that both enhancement of figures and the suppression of grounds are likely responsible for figure-related benefits in visual processing.

\section{General discussion}

Our results demonstrate that when targets appear within regions assigned figural status, they are discriminated consistently more accurately than when they appear within ground regions. Additionally, when figure-ground and figurally neutral displays were both presented (Exp. 3), targets appearing on figures were discriminated more accurately than those on either neutral or ground regions, whereas targets appearing on grounds were discriminated less accurately than those on either neutral or figure regions. We hypothesize that the increased discriminability of targets in figural regions may arise from an enhanced neural representation of figures, whereas the decreased discriminability of targets appearing on ground regions may arise from a suppressed neural representation of targets appearing within these regions. Such a view is consistent with previous primate neurophysiological studies (Lamme, 1995; Marcus \& Van Essen, 2002; Qiu et al., 2007) and with models of figure-ground assignment (e.g., Craft et al., 2007; Roelfsema et al., 2002; Vecera 2000; Vecera \& O'Reilly, 1998, 2000).

Given the close relationship between figure-ground assignment and attention, the sensory-gain control accounts used to explain attentional effects (e.g., Hawkins et al., 1990; Hillyard, Vogel, \& Luck, 1998) can be readily adapted to explain the increased saliency of figures following figureground assignment. It is possible that the phenomenological advantage of figures described by Rubin (1915/1958) and others may result from the enhancement of regions assigned as figure and the suppression of regions assigned as ground; in other words, the sensory gain for figures is "turned up," whereas the gain for grounds is "turned down." However, our results suggest that this gain control is independent of attention, because in our task observers' attention was directed to each region on the basis of its color and not of its status as a figure or a ground. Because the region containing the target was manipulated orthogonally to figural status, and because the attentional set for the color of the target-containing region should have strongly biased attention toward that region (cf. Folk et al., 1992), the differences we observed in discrimination accuracy for figures and grounds cannot be attributed solely to attention-related enhancement effects. This also suggests that figure-ground assignment can proceed independently of the focus of attention, consistent with reports that figure-ground assignment can occur preattentively and without attention resources (Driver, Baylis \& Rafal, 1992; Kimchi \& Peterson, 2008).

Consistent with this interpretation, a number of neurophysiological studies in nonhuman primates have shown that neuronal firing rates in response to figural regions are increased relative to firing rates in response to ground regions, even when those regions are outside the focus of attention. For instance, Lamme (1995) examined the responses of individual striate cortex (V1) neurons in macaque monkeys to figures versus grounds, as defined by the orientations of line segments in an array. When the segment that formed the figure region was located within a neuron's receptive field, he observed a $40 \%$ increase in firing rates as compared to when the receptive fields were located on the ground region. Similarly, Marcus and Van Essen (2002) showed an enhancement of responses in macaque V1 and V2 neurons when the figure fell within a cell's receptive field relative to when the ground fell within the receptive field. Importantly, Marcus and Van Essen manipulated the location of attention within the visual display. Responses to figure and ground regions were similar regardless of the location of attention, suggesting that perceptual enhancement and suppression effects were independent of those associated with attentional processes.

Finally, in a study by Qiu et al. (2007) monkeys were presented with overlapping figure-ground displays, designed such that one region of the display (the "figure") occluded the other region (the "ground"). They showed that the firing rates of V2 neurons whose receptive fields were located on the border between the two regions were increased following the assignment of border ownership to the occluding figure (a process analogous to figure-ground assignment), regardless of whether attention was directed to that region or the occluded ground region. Critically, Qiu et al. also demonstrated that 
when attention was directed to the occluding "figure" region there was further enhancement of neuronal responses in the V2 neurons of interest. The authors interpreted this result as providing evidence for an interactive account of object-based attentional selection, in which the V2 neurons involved in figure-ground segregation also provide an interface for topdown attentional selection processes. This supports the assertion that figure-ground assignment produces enhancement effects that precede, but may interact with, attentional enhancement processes.

Additionally, the perceptual enhancement following figure-ground assignment characterized in the present experiments and in Qiu et al.'s (2007) study may instead reflect an enhancement of well-defined shapes. In these studies, the ground was likely to be perceived as "shapeless," continuing behind the figure. In other words, the figure-ground displays contained a shared contour that was associated with the figure, resulting in a well-defined figure and an ambiguously shaped ground. Our experiments cannot determine whether or not a shared contour is required in order to observe a perceptual enhancement of figures. Instead, this effect might reflect a processing advantage for completed shapes and not for "shapeless" regions. Future experiments could modify the stimuli such that the figure-ground displays contained welldefined figures and grounds. If perceptual enhancement were found, it could be concluded that enhancement extends to all figures, and not simply to those juxtaposed with a "shapeless" ground.

As we noted in the introduction, Nelson and Palmer (2007) demonstrated both detection and discrimination benefits for targets appearing in figural regions over those appearing in ground regions, a result they interpreted as being due to attention-related enhancement processes. Specifically, they discussed their results as being due to a preferential allocation of attention to figures following figure-ground assignment, which led to attention-related processing benefits for targets falling within the figure. However, the results of our experiments, coupled with the neurophysiological studies outlined above, suggest that regions assigned figural status need not draw attention in order to receive processing benefits. This points toward the possibility that the results of Nelson and Palmer may be due to an interaction between enhancements due to figure-ground assignment and due to attention. A similar argument could be made for the present experiments, which cannot definitively rule out the possibility of an interaction between each of these enhancement effects. For example, figure-ground assignment might occur quickly enough for the figure to draw attention prior to shifting to the taskrelevant colored region. However, the neurophysiological effects showing clear evidence of perceptual enhancement even in the absence of attention, coupled with the strong manipulation of feature-based attention used in the present work, suggest a role for perceptual enhancement in driving at least a portion of our observed figural processing benefits. Future experiments should explore the timescale over which each of these enhancement effects operates.

The notion of a bidirectional relationship between figureground assignment and attention is consistent with the results of Vecera et al. (2004), who found that when two regions of a figure-ground display are ambiguous with respect to figural status, directing exogenous attention to one of the regions influences which item is perceived as figure. They also showed that even when strong image-based cues to figureground assignment were present, the location of attention still affected figure-ground assignment (see also Cosman \& Vecera, 2010). This interaction of figure-ground assignment and attention fits well with theories and computational models of figure-ground assignment (e.g., Roelfsema et al., 2002; Vecera, 2000; Vecera \& O'Reilly, 1998). For example, in Vecera's (2000) "biased-competition" model of figureground assignment, the perceptual representations of figures are more "active" than those of grounds, causing figures to compete for visual processing resources more effectively. This increased activity can result either from image-based cues to figure-ground assignment, such as the convexity cues used in our study, or from top-down influences of object recognition or attention processes. The results of the present study suggest that following figure-ground assignment, an early enhancement of figures can serve to bias the allocation of visual resources such as attention or memory to particular items in a scene.

Taken together, our results suggest that objects assigned figural status have an enhanced perceptual representation over ground regions, underscoring the complex relationship between figure-ground assignment and attention by showing that these processes interact closely to affect the perception of items in a scene. In the future, it will be important to better understand the nature of this relationship and the constraints that this interaction puts on the later perceptual processing of objects.

Author note This research was supported by NSF Grant No. BCS $03-$ 3911, awarded to S.P.V., and NIH Grant No. F32EY023922-01, awarded to J.D.C. We thank Rolf Nelson and two anonymous reviewers for their constructive feedback on an earlier version of this article.

\section{References}

Bahnsen, P. (1928). Eine Untersuchung über Symmetrie und Asymmetrie bei visuellen Wahrnehmungen. Zeitschrift für Psychologie, 108, $355-361$.

Baylis, G. C., \& Driver, J. (1993). Visual attention and objects: Evidence for hierarchical coding of location. Journal of Experimental Psychology: Human Perception and Performance, 19, 451-470. doi:10.1037/0096-1523.19.3.451

Carrasco, M. (2006). Covert attention increases contrast sensitivity: Psychophysical, neurophysiological and neuroimaging studies. 
Progress in Brain Research, 154, 33-70. doi:10.1016/S00796123(06)54003-8

Carrasco, M. (2011). Visual attention: The past 25 years. Vision Research, 51, 1484-1525. doi:10.1016/j.visres.2011.04.012

Cosman, J. D., \& Vecera, S. P. (2010). Attention affects visual perceptual processing near the hand. Psychological Science, 21, 1254-1258. doi: $10.1177 / 0956797610380697$

Craft, E., Schütze, H., Niebur, E., \& von der Heydt, R. (2007). A neural model of figure-ground organization. Journal of Neurophysiology, 97, 4310-4326. doi:10.1152/jn.00203.2007

Desimone, R., \& Duncan, J. (1995). Neural mechanisms of selective visual attention. Annual Review of Neuroscience, 18, 193-222. doi:10.1146/annurev.ne.18.030195.001205

Driver, J., \& Baylis, G. C. (1996). Edge-assignment and figure-ground segmentation in short-term visual matching. Cognitive Psychology, 31, 248-306. doi:10.1006/cogp.1996.0018

Driver, J., Baylis, G. C., \& Rafal, R. D. (1992). Preserved figure-ground segregation and symmetry perception in visual neglect. Nature, 360 , 73-75.

Dutton, M. B., \& Traill, P. M. (1933). A repetition of Rubin's figureground experiment. British Journal of Psychology, 23, 389-400.

Folk, C. L., Remington, R. W., \& Johnston, J. C. (1992). Involuntary covert orienting is contingent on attentional control settings. Journal of Experimental Psychology: Human Perception and Performance, 18, 1030-1044. doi:10.1037/0096-1523.18.4.1030

Gibson, B. S. (1994). Visual attention and objects: One versus two or convex versus concave? Journal of Experimental Psychology: Human Perception and Performance, 20, 203-207. doi:10.1037/ 0096-1523.20.1.203

Hawkins, H. L., Hillyard, S. A., Luck, S. J., Mouloua, M., Downing, C. J., \& Woodward, D. P. (1990). Visual attention modulates signal detectability. Journal of Experimental Psychology: Human Perception and Performance, 16, 802-811. doi:10.1037/00961523.16.4.802

Hecht, L. N., Spencer, J. P., \& Vecera, S. P. (2015). A dynamic neural field model of temporal order judgments. Journal of Experimental Psychology: Human Perception and Performance, 41, 1718-1733. doi:10.1037/xhp0000097

Hillyard, S. A., Vogel, E. K., \& Luck, S. J. (1998). Sensory gain control (amplification) as a mechanism of selective attention: Electrophysiological and neuroimaging evidence. Philosophical Transactions of the Royal Society B, 353, 1257-1270. doi:10. 1098/rstb.1998.0281

Kanizsa, G., \& Gerbino, W. (1976). Convexity and symmetry in figureground organization. In M. Henle (Ed.), Vision and artifact (pp. 25 32). New York, NY: Springer.

Kimchi, R., \& Peterson, M. A. (2008). Figure-ground segmentation can occur without attention. Psychological Science, 19, 660-668. doi: 10.1111/j.1467-9280.2008.02140.x

Koffka, K. (1935). Principles of Gestalt psychology. New York, NY: Harcourt, Brace.

Lamme, V. A. F. (1995). The neurophysiology of figure-ground segregation in primary visual cortex. Journal of Neuroscience, 15, 16051615.

Lester, B. D., Hecht, L. N., \& Vecera, S. P. (2009). Visual prior entry for foreground figures. Psychonomic Bulletin \& Review, 16, 654-659. doi:10.3758/PBR.16.4.654

Marcus, D. S., \& Van Essen, D. C. (2002). Scene segmentation and attention in primate cortical areas V1 and V2. Journal of Neurophysiology, 88, 2648-2658.

Nelson, R. A., \& Palmer, S. E. (2007). Familiar shapes attract attention in figure-ground displays. Perception \& Psychophysics, 69, 382-392. doi:10.3758/BF03193759
Palmer, S. E. (1999). Gestalt perception. In R. A. Wilson \& F. C. Keil (Eds.), The MIT encyclopedia of the cognitive sciences (pp. 344346). Cambridge, MA: MIT Press.

Palmer, S. E. (2002). Perceptual grouping: It's later than you think. Current Directions in Psychological Science, 11, 101-106.

Peterson, M. A. (1994). Object recognition processes can and do operate before figure-ground organization. Current Directions in Psychological Science, 3, 105-111. doi:10.1111/1467-8721. ep10770552

Peterson, M. A., \& Gibson, B. S. (1993). Shape recognition inputs to figure-ground organization in three dimensional displays. Cognitive Psychology, 25, 383-429.

Peterson, M. A., \& Skow, E. (2008). Inhibitory competition between shape properties in figure-ground perception. Journal of Experimental Psychology: Human Perception and Performance, 34, 251-267. doi:10.1037/0096-1523.34.2.251

Pomerantz, J. R., \& Kubovy, M. (1986). Theoretical approaches to perceptual organization: Simplicity and likelihood principles. In K. R. Boff, L. Kaufman, \& J. P. Thomas (Eds.), Handbook of perception and human performance: Vol. 2. Cognitive processes and performance (pp. 1-46). New York, NY: Wiley.

Qiu, F. T., Sugihara, T., \& von der Heydt, R. (2007). Figure-ground mechanisms provide structure for selective attention. Nature Neuroscience, 10, 1492-1499.

Rock, I. (1975). An introduction to perception. New York, NY: Macmillan.

Rock, I., \& Kremen, I. (1957). A re-examination of Rubin's figural aftereffect. Journal of Experimental Psychology, 53, 23-30.

Roelfsema, P. R., Lamme, V. A. F., Spekreijse, H., \& Bosch, H. (2002). Figure-ground segregation in a recurrent network architecture. Journal of Cognitive Neuroscience, 14, 525-537. doi:10.1162/ 08989290260045756

Rubin, E. (1958). Figure and ground. In D. C. Beardslee \& M. Wertheimer (Eds.), Readings in perception (pp. 194-203). Princeton, NJ: Van Nostrand (Original work published 1915).

Salvagio, E., Cacciamani, L., \& Peterson, M. A. (2012). Competitionstrength-dependent ground suppression in figure-ground perception. Attention, Perception, \& Psychophysics, 74, 964-978. doi:10. 3758/s13414-012-0280-5

Vecera, S. P. (2000). Toward a biased competition account of objectbased segregation and attention. Brain and Mind, 1, 353-384.

Vecera, S. P., Flevaris, A. V., \& Filapek, J. C. (2004). Exogenous spatial attention influences figure-ground assignment. Psychological Science, 15, 20-26. doi:10.1111/j.0963-7214.2004.01501004.x

Vecera, S. P., \& O'Reilly, R. C. (1998). Figure-ground organization and object recognition processes: An interactive account. Journal of Experimental Psychology: Human Perception and Performance, 24, 441-462. doi:10.1037/0096-1523.24.2.441

Vecera, S. P., \& O'Reilly, R. C. (2000). Graded effects in hierarchical figure-ground organization: Reply to Peterson (1999). Journal of Experimental Psychology: Human Perception and Performance, 26, 1221-1231. doi:10.1037/0096-1523.26.3.1221

Vecera, S. P., Vogel, E. K., \& Woodman, G. F. (2002). Lower region: A new cue for figure-ground assignment. Journal of Experimental Psychology: General, 131, 194-205. doi:10.1037/0096-3445.131. 2.194

Wagemans, J., Elder, J. H., Kubovy, M., Palmer, S. E., Peterson, M. A., Singh, M., \& von der Heydt, R. (2012). A century of Gestalt psychology in visual perception: I. Perceptual grouping and figureground organization. Psychological Bulletin, 138, 1172-1217. doi: $10.1037 / \mathrm{a} 0029333$

Wong, E., \& Weisstein, N. (1982). A new perceptual context-superiority effect: Line segments are more visible against a figure than against a ground. Science, 218, 587-589. 